\title{
Occult Fallopian Tube Carcinoma Detected in Routine Pelvic Washing Specimens Submitted for Staging: Another Justification for Pelvic Washing Cytology?
}

Julie M. Jorns, M.D. ${ }^{*}$ and Stewart M. Knoepp, M.D., Ph.D.

Peritoneal washing cytology is important in the staging, prognosis, and treatment schema for women diagnosed with gynecologic malignancies. Additionally, peritoneal washings are often performed before the specific diagnosis or even the distinction between benign or malignant lesion is known. Occasionally, the cytology and surgical pathology specimens, although obtained during the same procedure, will show unexpected and/or discordant findings. Perhaps, the greatest dilemma occurs when atypical cells suggestive of malignancy are identified in peritoneal washing specimens when a corresponding benign or discordantly low grade malignant diagnosis is made in the surgical pathology specimen. We present two such cases in which further investigation proved extremely valuable, not only leading to resolution of initially discrepant cytologic and surgical pathology findings but also in the process leading to second diagnoses of early fallopian tube carcinoma. Increasing evidence appears to be pointing to the fallopian tube as the site of origin of many serous carcinomas previously thought to be of primary pelvic or ovarian origin. These cases further confirm the utility of peritoneal washing cytology as a diagnostic modality when routine gross examination and sampling of the surgical specimens alone would have failed to provide the complete diagnosis. Diagn. Cytopathol. 2009;37:923-929. @ 2009 Wiley-Liss, Inc.

Key Words: pelvic washing; cytology; fallopian tube; carcinoma

Department of Pathology, University of Michigan, Ann Arbor, Michigan

*Correspondence to: Julie M. Jorns, M.D., Department of Pathology, University of Michigan, Medical Sciences I, 1301 Catherine St, Ann Arbor, MI 48109. E-mail: jjorns@umich.edu

Received 26 February 2009; Accepted 12 May 2009

DOI $10.1002 / \mathrm{dc} .21129$

Published online 16 September 2009 in Wiley InterScience (www. interscience.wiley.com).
The practice of performing peritoneal washing cytology in cases of suspected gynecologic malignancy was introduced by Keetal and Elkins in 1965 in attempts to detect early cancer spread ${ }^{1}$ and was subsequently shown to have significant prognostic implications. ${ }^{2}$ This led to widespread acceptance and utilization with incorporation into the International Federation of Gynecology and Obstetrics (FIGO) staging classification of ovarian neoplasms in 1975, and endometrial carcinomas in 1989, and is still the foundation by which women get stratified into different treatment approaches. This is likely the reason that many institutions have a policy of performing peritoneal washing cytology in the vast majority of gynecologic surgeries, both benign and malignant, whether a preoperative diagnosis has been made or not. Occasionally, however, cytology may show unexpected findings. We present two such cases in which peritoneal washing cytology results conflicted with those of the surgical pathology examination, which posed a significant diagnostic dilemma. However, both cases also demonstrate how further investigation helped to resolve this dilemma and further confirm the utility of peritoneal washing cytology in the diagnosis of gynecologic malignancies.

\section{Case 1}

A 78-year-old gravida 4, para 4 postmenopausal female presented to her primary care physician complaining of hematuria. An extensive urologic work-up including urine cytology, cystoscopy with bladder biopsies, and retrograde pyelogram was negative. However, she later noted a pink vaginal discharge and a pelvic ultrasound revealed a slightly thickened endometrial stripe $(4 \mathrm{~mm})$. She subse- 
quently underwent endometrial biopsy which showed welldifferentiated endometrial adenocarcinoma, endometrioid type, preliminarily FIGO grade 1 . She was deemed to have clinical stage 1 endometrial adenocarcinoma and underwent Da Vinci-assisted total laparoscopic hysterectomy (LH), bilateral salpingo-oopherectomy, bilateral pelvic, obturator, and periaortic lymphadenectomy, and pelvic washings.

The main surgical resection specimen demonstrated a $3.2 \times 2.5 \times 0.7 \mathrm{~cm}$ grossly myoinvasive white-tan tumor involving the posterior uterus and portion of the anterior uterus without involvement of the lower uterine segment or cervix. Histologic examination revealed a well-differentiated but complex glandular architecture with areas of confluence and associated desmoplastic stroma. The glands showed enlarged, multilayered epithelial cells with clumped chromatin, prominent nucleoli and interspersed mitotic figures (Fig. 1). Neither a significant solid component nor areas of divergent differentiation were identified. These features were diagnostic for a FIGO grade 1 endometrioid endometrial adenocarcinoma, and consistent with the prior diagnosis made from a curettage specimen. The tumor displayed myoinvasion into the inner half of the myometrium $(0.4 \mathrm{~cm}$ maximum invasion $/ 1.3 \mathrm{~cm}$ total uterine thickness), and no true angiolymphatic invasion was identified. Interestingly, pseudo-vascular space involvement (VSI) was seen in large, thick-walled ectatic vessels in the outer myometrium. This phenomenon was recently described by Kithara et al., ${ }^{3}$ and consists of artificially detached fragments of friable tumor that become embedded in myometrial spaces during grossing procedures. It is more commonly seen in mechanically manipulated $\mathrm{LH}$ specimens, and tumor foci lack features of true VSI such as adherence to the vessel walls, tumor molding in the shape of the associated vessel, and/or tumor thrombi. Nevertheless, the findings provided an additional source of consideration for origin of the atypical cells (discussed below) seen in the pelvic washing. The recognition of such detached tumor fragments in lymphovascular spaces as artifactual is important in preventing overtreatment of patients with otherwise low-grade, low-stage tumors. Additional findings included medial calcific atherosclerosis of uterine arterioles, benign paratubal cysts, bilaterally, unremarkable ovaries, and mild reactive atypia of bilateral fallopian tubes. A total of 37 lymph nodes were identified, all of which were negative for malignancy. On the basis of the surgical/pathologic findings, the patient's endometrial adenocarcinoma was designated as stage IB.

Despite the relatively favorable diagnosis, concurrent peritoneal washing cytology showed the liquid-based preparations to contain scattered highly atypical cells with high nuclear:cytoplasmic $(\mathrm{N}: \mathrm{C})$ ratio, coarse chromatin, and prominent nucleoli (Fig. 2). These cells were scant in the cell block and thus could not be further classified via immunohistochemical staining. Also, present within both the liquid-based and cell block specimens were scattered benign-appearing tubal epithelial cells, some of which displayed a spectrum of reactive atypia (Fig. 3). The apparent disparity between the findings seen in the surgical pathology and cytology specimens posed a significant diagnostic dilemma, especially due to the scant nature of the malignant-appearing cells within the pelvic washings. Because of this, the surgical specimen was re-examined and although no other significant gross findings were identified the fallopian tubes were submitted entirely for histologic evaluation, revealing a $0.3 \mathrm{~cm}$ focus of high-grade serous carcinoma with cytologic features identical to that of the scattered malignant cells seen in the pelvic washings (Fig. 4). The carcinoma appeared to arise in the fimbriated end of the fallopian tube and in direct association with in situ carcinoma, which was highlighted by p53 immunohistochemical staining. Thus, the patient was given the second diagnosis of a high risk stage 1 fallopian tube carcinoma (stage 1c with tumor confined to the tube but with positive pelvic washings). Completion staging including omentectomy, peritoneal biopsies, and bilateral diaphragm scrapings was negative. At the time of this report she had just began the first of four planned cycles with Carboplatin and Taxol chemotherapy.

\section{Case 2}

A 66-year-old gravida 3, para 3, postmenopausal female presented to the University of Michigan alternative hysterectomy clinic for second opinion for treatment of her "fibroid uterus." She had brought a copy of a pelvic ultrasound from 1999, which demonstrated a $7 \mathrm{~cm}$ solid mass posterior to the uterus. The patient then underwent a pelvic Magnetic Resonance Imaging (MRI) which revealed a $20 \times 12 \mathrm{~cm}$ solid mass with focal degenerative changes located posterior to the uterus without clear identification of the organ of origin. The patient consented to have the mass resected but desired removal of the least amount of tissue possible only. At the time of surgery, the mass was found to arise from the left ovary with grossly unremarkable uterus and right adnexa. Unilateral oopherectomy with partial salpingectomy was performed.

The surgical resection specimen consisted of a $1.55 \mathrm{~kg}$, $22 \times 14 \times 9.5 \mathrm{~cm}$ ovary completely replaced by a predominantly firm, white, fibrous tumor with foci of yellow coloration and cystic change and an attached $8 \mathrm{~cm}$ portion of adhesed fallopian tube. Microscopic examination revealed the tumor to consist predominantly of intersecting bundles of banal spindle cells and dense hyaline bands with interspersed edematous regions containing hyaline droplets (Fig. 5). The minority of the tumor which corresponded to the grossly yellow areas was slightly more cellular with banal, plump ovoid cells, which stained positive for oil-red-O and weakly for inhibin- $\alpha$. There was no cytologic atypia and mitoses were not iden- 


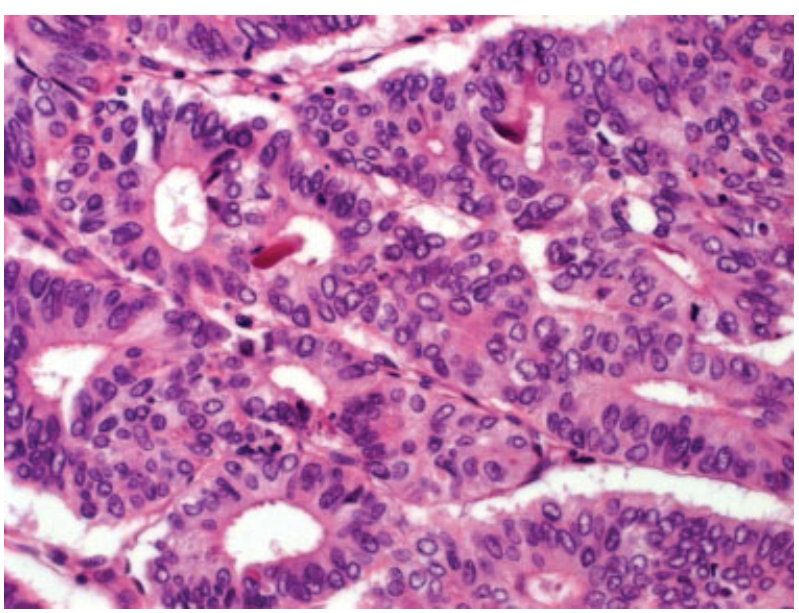

Fig. 1. Case 1: Well-differentiated endometrial adenocarcinoma, endometrioid type. [Color figure can be viewed in the online issue, which is available at www.interscience.wiley.com.]

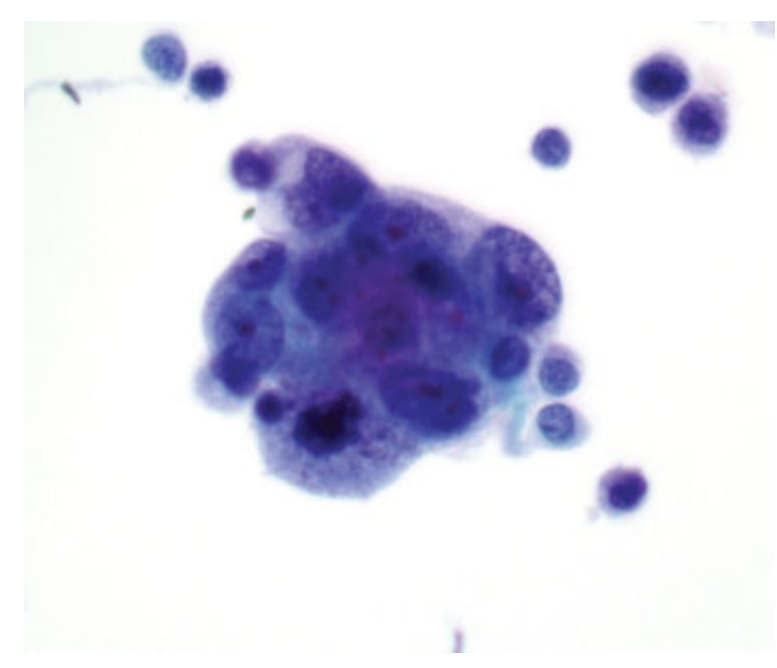

Fig. 2. Case 1: Pelvic washing cytology with highly atypical cells. [Color figure can be viewed in the online issue, which is available at www.interscience.wiley.com.]

tified. A reticulin stain showed characteristic encircling of the individual tumor cells. On the basis of these findings, the patient was diagnosed with fibrothecoma.

Despite the apparent benign diagnosis, concurrent pelvic washings showed three-dimensional clusters of highly atypical cells with increased $\mathrm{N}: \mathrm{C}$ ratio, coarse chromatin and prominent nucleoli (Fig. 6, Top Panel). These cells were also prominent in the cell block in which they also displayed intracytoplasmic vacuoles and lumen formation, findings all consistent with adenocarcinoma (Fig. 6, Bottom Panel). This disparity led to further investigation including review of the surgical pathology slides and immunohistochemical staining of the cell block. Upon review, a single slide of fallopian tube revealed mild cytologic atypia, thus the remainder of the fallopian tube

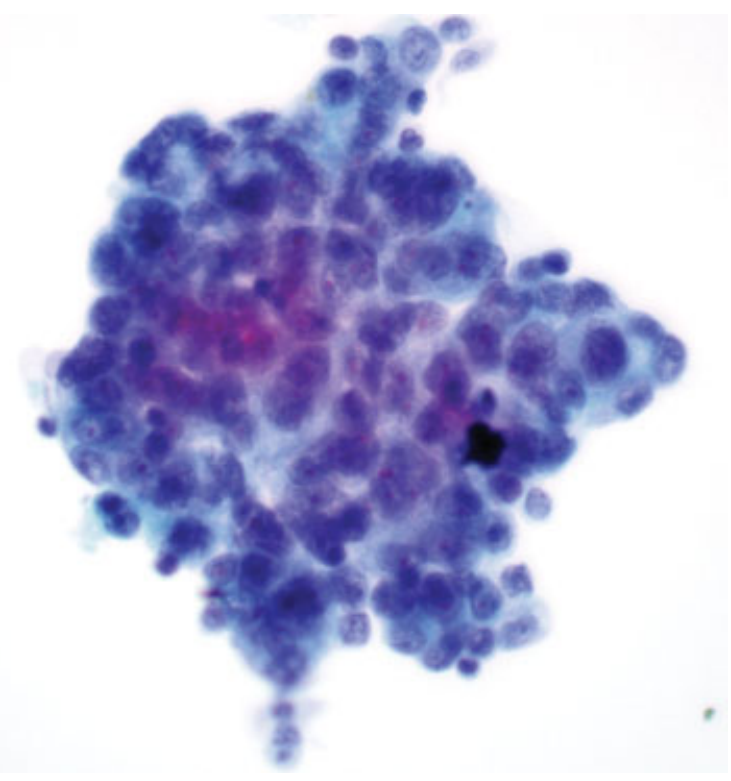

Fig. 3. Case 1: Pelvic washing cytology with tubal epithelial cells displaying a spectrum of reactive atypia. [Color figure can be viewed in the online issue, which is available at www.interscience.wiley.com.]

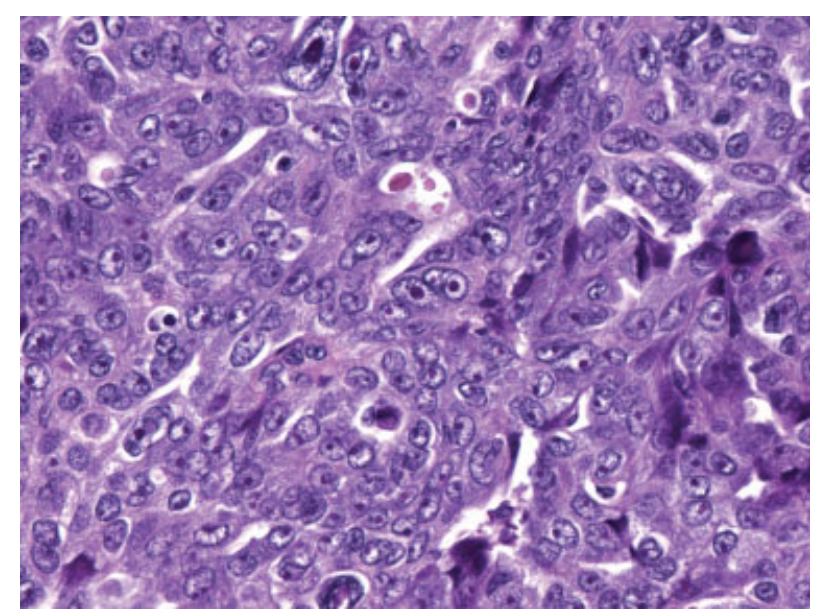

Fig. 4. Case 1: Fallopian tube with high-grade serous carcinoma. [Color figure can be viewed in the online issue, which is available at www. interscience.wiley.com.]

was submitted for histologic evaluation and deeper sections and immunohistochemical stains were performed on this tissue block. None of the additional sections of fallopian tube revealed foci of atypia; however, deeper sections of the slide with fallopian tube atypia revealed loss of normal epithelial stratification, large, hyperchromatic cells with increased $\mathrm{N}$ : $\mathrm{C}$ ratio and prominent nucleoli and interspersed mitotic figures, consistent with intraepithelial carcinoma (Fig. 7). These cells showed strong p53 expression and an increased proliferation index via Mib-1 (ki67) immunohistochemical staining. Immunostains performed on the pelvic washing cell block showed positive staining of the malignant cells with cytokeratin 7 , epithe- 


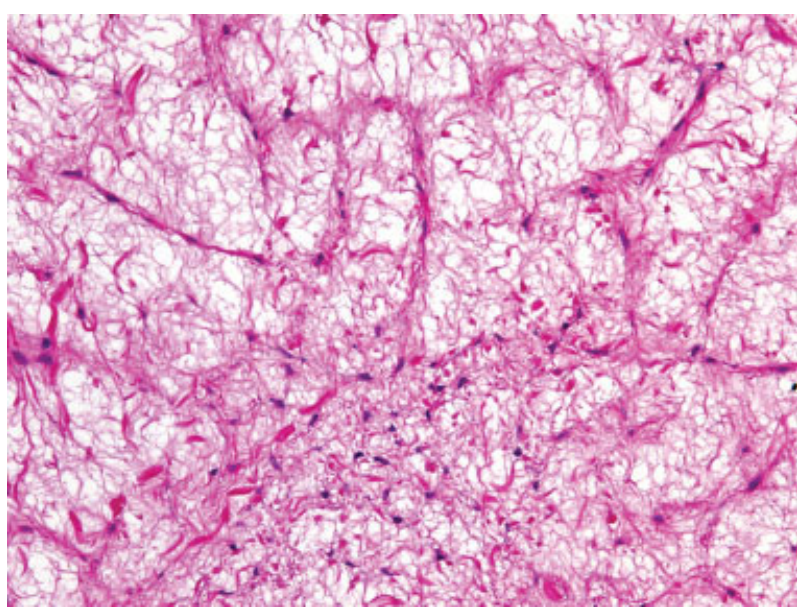

Fig. 5. Case 2: Ovarian fibrothecoma. [Color figure can be viewed in the online issue, which is available at www.interscience.wiley.com.]
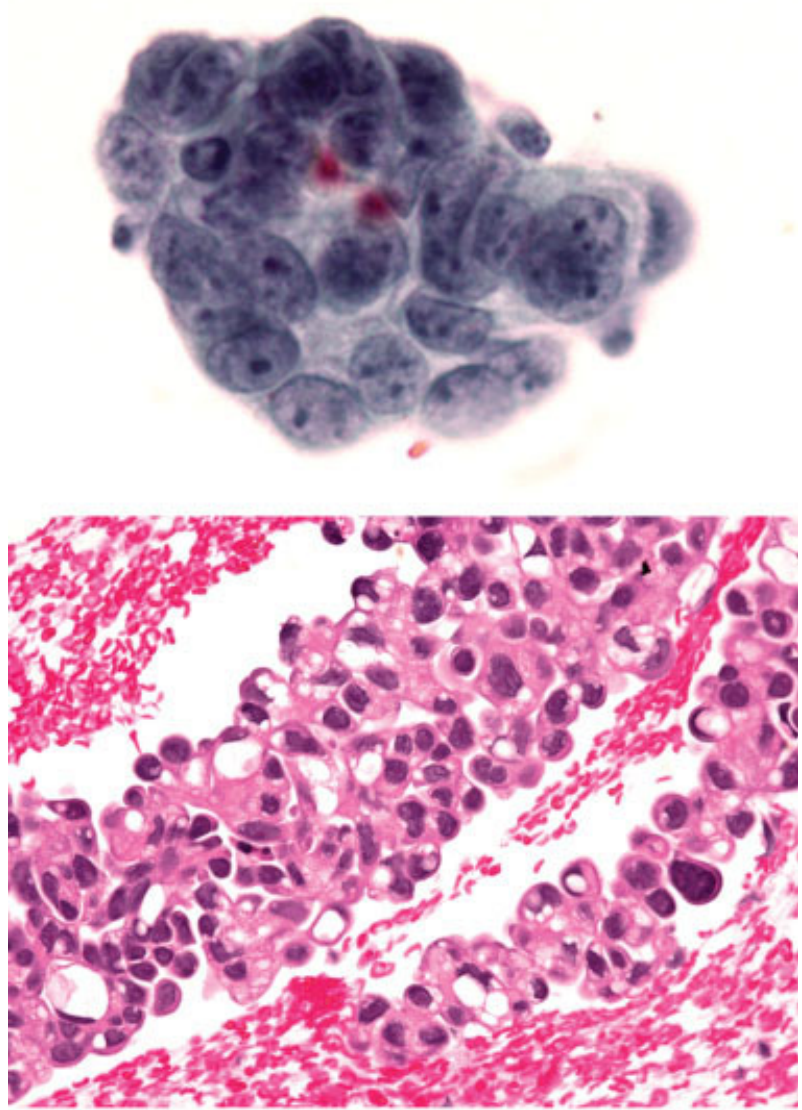

Fig. 6. Case 2: Pelvic washing cytology with highly atypical cell clusters in liquid-based preparations (top panel) and cell block (bottom panel). [Color figure can be viewed in the online issue, which is available at www.interscience.wiley.com.]

lial membrane antigen (EMA), Ber-EP4, and p53 and no staining with CEA or calretinin, an immunophenotype consistent with serous carcinoma. It was recommended

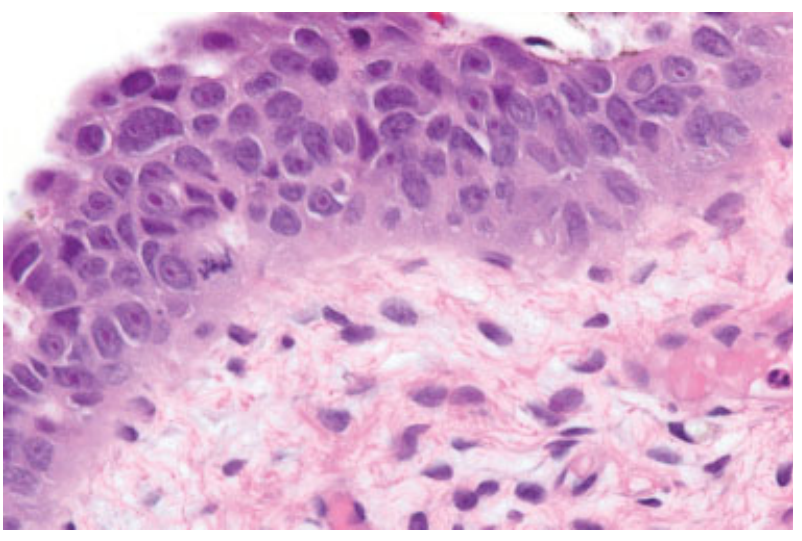

Fig. 7. Case 2: Fallopian tube carcinoma in situ. [Color figure can be viewed in the online issue, which is available at www.interscience. wiley.com.]

that the patient undergo completion staging and possible chemotherapy but the patient declined, stating she was going to seek a second opinion. No further follow-up information was available at the time of this report.

\section{Discussion}

Peritoneal washing cytology is an important and accepted diagnostic procedure in the staging of ovarian and endometrial neoplasms. Many studies have examined the sensitivity of peritoneal washings and have shown variable results, with sensitivities ranging from as low as $50.7 \%$ to as high as $88 \% .^{4-7}$ This range likely reflects a variety of factors from clinical aspects such as organ of tumor origin, extent of peritoneal involvement, and histologic subtype to technical factors involved in the acquisition and preparation of the cytology specimen. Differences in study design and especially in selection criteria also affect sensitivity, with higher numbers seen in studies selecting for only those cases with histologically-proven disease. ${ }^{4}$

In contrast to the moderate sensitivity seen in pelvic washing specimens, the specificity of peritoneal washings is excellent $(\geq 98 \%)$, especially with biopsy confirmed intra-peritoneal cancer involvement. ${ }^{4}$ Occasional false positives have been well-documented, however, most notably in cases of endometriosis, endosalpingiosis, and reactive mesothelial atypia. ${ }^{8-10}$ The combination of both cytomorphologic findings, including the specimen background appearance, along with the clinical history is often sufficient to make the distinction from carcinoma. More specifically, the presence of both glandular and stromal cells with background hemosiderin-laden macrophages would point to the benign diagnosis of endometriosis as would the presence of ciliated cells in endosalpingiosis. Reactive mesothelial cells can form tight clusters with nuclear features that may mimic borderline or low grade ovarian carcinomas, especially when psammoma bodies are also present. Immunohistochemical stains can serve as an important 
adjunct in these cases that prove more challenging when differentiating between reactive mesothelial proliferations and adenocarcinoma, given that the cell block contains an adequate representation of the atypical cells. ${ }^{11,12}$

Despite the need to be cautious of these potential pitfalls most positive peritoneal washings are indicative of peritoneal involvement by the patient's known gynecologic malignancy. Up-staging of the neoplasm may be based solely upon peritoneal washing cytology, with prognostic implications and specific staging dependent on the organ of origin. In these cases, a positive peritoneal washing in patients with ovarian neoplasms have been found to display a generally poorer prognosis, ${ }^{5}$ but those with endometrial neoplasms appear to have little additional adverse affect on overall outcome, despite the potentially greater change in tumor stage. ${ }^{13-15}$ In fact, some have suggested that the presence of endometrial carcinoma cells in peritoneal washings of patients with low clinical stage disease are transient and may not be capable of metastatic growth and instead of representing intra-peritoneal spread likely are the result of surgical manipulation or prior hysteroscopy. ${ }^{16,17}$ Because of this there has some debate about the utility of peritoneal washings in clinically early stage endometrial carcinoma. These patients designated as surgical stage IIIA disease based on pelvic washings alone will also have similarly favorable outcomes with or without adjuvant treatment, thus its use is also controversial. ${ }^{17}$ Given these data, our findings are especially important because they provide an additional benefit to pelvic washing cytology (i.e., the initial identification of a tubal carcinoma may result from a positive pelvic washing cytology).

At first glance, case 1 appeared to be such a case in which the scattered atypical cells could represent dissemination of endometrial carcinoma in the peritoneal cavity, which may or may not have influenced further prognosis and therapy. These atypical cells were scant and accompanied by a significant population of reactive-appearing ciliated cells resembling fallopian tube epithelium, raising the question that this could be a reactive but benign process. However, the degree of atypia was higher than that seen in the patient's well-differentiated endometrial carcinoma, and the morphology was consistent with a serous tumor, raising the suspicion of a second occult malignancy. Further investigation with immunohistochemical stains could not be performed due to the paucity of atypical cells in the cell block but further evaluation of the surgical pathology specimen revealed a small focus of high-grade serous carcinoma of the distal fallopian tube, finally explaining the source of these scant but malignant cells in the peritoneal washings.

In case 2, the initial cytology and surgical pathology diagnoses were straightforward but clearly discordant. After ruling out error in specimen processing it was suspected that this patient also had a second occult malig- nancy. Immunohistochemical stains pointed to a serous carcinoma and further evaluation of the fallopian tube revealed an intraepithelial carcinoma. Despite negative imaging studies and the lack of gross findings noted at surgery, without completion staging we cannot speculate on the extent of disease in the contralateral fallopian tube and ovary or of the peritoneal cavity. Both this and the previous case, however, illustrate how follow-up of unexpected findings in peritoneal washing cytology led to second diagnoses of fallopian tube carcinoma which has significant prognostic and treatment implications. Although there is evidence to support a more favorable outcome there is still little known about the prognosis of these early fallopian tube carcinomas. ${ }^{18}$ However, based on a surgical stage of $1 \mathrm{C}$, current recommendations would have a patient receive platinum-based systemic chemotherapy, which would have been unlikely to occur in case 1 and would not have even been considered in case 2. ${ }^{19,20}$

Historically, primary fallopian tube carcinomas have been thought to be rare, making up only $0.3-1.1 \%$ of all gynecologic malignancies and with tubal intraepithelial carcinomas only accounting for rare cases. ${ }^{21}$ Recent evidence, however, has pointed to the fallopian tube as the source of many more high-grade serous carcinomas than previously thought. This discovery has come about due to the practice of prophylactic bilateral salpingo-oophorectomy (BSO) in patients with BRCA1 and BRCA2 gene mutations who have a marked increased susceptibility for breast and ovarian cancers. ${ }^{22-27}$ Prophylactic BSO has been shown to decrease these risks significantly, with a $96 \%$ risk reduction for ovarian carcinoma ${ }^{28}$ as well as $56 \%$ and $46 \%$ breast cancer risk reduction for BRCA1 and BRCA2 mutation carriers, respectively. ${ }^{29}$ Additionally, investigators noted that a small number of these specimens harbored occult cancers with a much higher than expected incidence of early fallopian tube cancers. This led to protocols designed to thinly section the ovaries and fallopian tubes for maximum identification of these in situ and early invasive cancers. ${ }^{22-24}$ These rigorous grossing techniques have appeared to implicate the distal (fimbriated) end as the predominant site of tumor origin and have led to a new model for cancer development as described by Crum et al., which describes the evolution from genotoxic stress leading to p53 mutations followed by rapid progression from intra-epithelial carcinoma to widely disseminated high-grade serous carcinoma. Using this protocol, these investigators also examined the fallopian tubes of a small series of patients with non-familial pelvic serous carcinomas and noted that the majority involved the endosalpinx and more than half had evidence of early cancer arising in the fallopian tube, suggesting that it is likely the source of many high-grade serous carcinomas previously thought to be primary peritoneal in origin and is likely not limited to patients with 
increased genetic susceptibility. It has also been suggested that many high-grade serous carcinomas classified as originating in the ovary may also be of distal fallopian tube origin. Because of their rapid progression, these tumors would present at higher stage in non-BRCA carriers (not undergoing prophylactic BSO) and due to the proximity to the ovary would more likely present as a tubo-ovarian complex. Standard grossing techniques only include limited sampling of the fallopian tube and thus an associated in situ component which would suggest a possible fallopian tube origin could easily be missed. ${ }^{24,25}$ Similar gene expression profiles between these serous carcinomas and benign tubal epithelium provides further evidence to support this theory. ${ }^{30}$

The utility of peritoneal washing cytology in the identification of occult malignancy has been well-documented, and thus, its use is recommended at the time of prophylactic BSO in BRCA mutation carriers who are at greater risk. $^{31}$ Its use in presumed benign disease, however, has been of great debate as some studies have shown its use to be of too low utility and high cost to justify processing of all specimens. ${ }^{32}$ However, many institutions, including ours, perform, process, and examine peritoneal washings in the vast majority of gynecologic surgeries, both benign and malignant. This is frequently independent of whether a preoperative diagnosis has been rendered. We acknowledge that cost-saving measures are important in the laboratory but would argue strongly that enhanced patient care outweighs the modest additional cost with regard to pelvic washing cytology. The cases presented herein lend further support to an enhanced benefit/cost ratio, in that the two patients were not known to be BRCA mutation carriers or to have a strong family history of breast and/or ovarian cancer but were found to have early fallopian tube carcinomas which changed their prognosis as well as their treatment recommendations. Although the cases we present may be unusual and infrequent, the end result was that for these two patients, and potentially others, this would not have occurred without evaluation of the peritoneal washing specimens. Additionally, these cases illustrate how correlation between peritoneal washing cytology and surgical pathology findings were crucial to the discovery of occult malignancy. Thus, although cytopathologists must be aware of the potential pitfalls that could lead to a false-positive diagnosis in peritoneal washing cytology specimens, it is important to stress the utility of further investigation and ancillary studies when faced with discrepancies between cytology and surgical pathology findings.

\section{References}

1. Keetal WC, Elkins HB. Experience with radioactive colloidal gold in the treatment of ovarian cancer. Am J Obstet Gynecol 1956;71: $553-568$.
2. Creasman WT, Rutledge F. The prognostic value of peritoneal cytology in gynecologic malignant disease. Am J Obstet Gynecol 1971;110:773-781.

3. Kithara S, Walsh C, Frumovitz M, Malpica A, Silva EG. Vascular pseudoinvasion in laparoscopic hysterectomy specimens for endometrial carcinoma: A grossing artifact? Am J Surg Pathol 2009;33: 298-303.

4. Fadare O, Mariappan MR, Wang S, Hileeto D, McAlpine J, Rimm DL. The histologic subtype of ovarian tumors affects the detection rate by pelvic washings. Cancer Cytopathol 2004;102:150-156.

5. Zuna RE, Behrens A. Peritoneal washing cytology in gynecologic cancers: Long-term follow-up of 355 patients. J Natl Cancer Inst 1996;88:980-987.

6. Zuna RE, Mitchell ML, Mullick DA, Weijchert WM. Cytohistologic correlation of peritoneal washing cytology in gynecologic disease. Acta Cytol 1989;33:327-335.

7. Lowe E, McKenna H. Peritoneal washing cytology: A retrospective analysis of 175 gynaecologic patients. Aust N Z J Obstet Gynaecol 1989;29:55-61.

8. Markuly SN, Fanning J, Hindman TL, Galle PC, Ertmoed E, Hilgers RD. False-positive malignant peritoneal cytology associated with pelvic endometriosis. J Am Osteopath Assoc 1994;94:157-161.

9. Covell JL, Carry JB, Feldman PS. Peritoneal washings in ovarian tumors: Potential sources of error in cytologic diagnosis. Acta Cytol 1985;29:310-316.

10. Weir MM, Bell DA. Cytologic identification of serous neoplasms in peritoneal fluids. Cancer 2001;93:309-318.

11. Shield P. Peritoneal washing cytology. Cytopathology 2004;15:131141.

12. Selvaggi SM. Diagnostic pitfalls of peritoneal washing cytology and the role of cell blocks in their diagnosis. Diagn Cytopathol 2003;28:335-341.

13. Soper JT, Johnson P, Johnson V, Berchuck A, Clarke-Pearson DL. Comprehensive restaging laparotomy in women with apparent early ovarian carcinoma. Obstet Gynecol 1992;80:949-953.

14. Fadare O, Mariappan MR, Hileeto D, Wang S, McAlpine JN, Rimm DL. Upstaging based solely on positive peritoneal washing does not affect outcome in endometrial cancer. Mod Pathol 2005; 18:673-680.

15. Tebeu PM, Popowski Y, Verkooijen HM, et al. Positive peritoneal cytology in early-stage endometrial cancer does not influence prognosis. Br J Cancer 2004;91:720-724.

16. Hirai Y, Takeshima N, Kato T, Haasumi K. Malignant potential of positive peritoneal cytology in endometrial cancer. Obstet Gynecol 2001;97:725-728.

17. Obermair A, Geramou M, Gucer F, et al. Does hysteroscopy facilitate tumor cell dissemination? Incidence of peritoneal cytology from patients with early stage endometrial carcinoma following dilation and curettage (D\&C) versus hysteroscopy and D\&C. Cancer 2000;88:139-143.

18. Carlson JW, Miron A, Jarboe EA, et al. Serous tubal intraepithelial carcinoma: Its potential role in primary peritoneal serous carcinoma and serous cancer prevention. J Clin Oncol 2008;26:4160-4165.

19. Park CK, Apte S, Acs G, Harris EER. Abeloff's clinical oncology. In: Abeloff MD, Armitage JO, Niederhuber JE, Kastan MB, McKenna WG, editors. Cancer of the endometrium. 4th ed. Chapter 92, Philadelphia, PA: Churchill Livingstone Elsevier, 2008.

20. Katz VL, Dotters DJ. Neoplastic diseases of the fallopian tubes: Screening, benign and malignant epithelial and germ cell neoplasms, sex-cord stromal tumors. In: Katz VL, Lentz G, Lobo RA, Gershenson D, editors. Katz comprehensive gynecology. 5th ed. Chapter 34, Philadelphia, PA: Mosby Elsevier, 2007.

21. Alvarado-Cabrero I, Cheung A, Radcuff A. World Health Organization classification of tumors (pathology and genetics): Tumors of the breast and female genital organs. In: Tavassoli FA, Devilee P, editors. Chapter 3, Tumors of the fallopian tube. Lyon, France: IARC Press, 2003. p 203-209. 


\section{OCCULT FALLOPIAN TUBE CARCINOMA}

22. Powell CB, Kenley E, Chen LM, et al. Risk-reducing salpingooophorectomy in BRCA mutation carriers: Role of serial sectioning in the detection of occult malignancy. J Clin Oncol 2005;23:127132.

23. Finch A, Shaw P, Rosen B, Murphy J, Narod SA, Colgan TJ. Clinical and pathologic findings of prophylactic salpingo-oophorectomies in 159 BRCA1 and BRCA2 carriers. Gynecol Oncol 2006;100:58-64.

24. Crum CP, Drapkin R, Kindelberger D, Medeiros F, Miron A, Lee Y. Lessons from BRCA: The tubal fimbria emerges as an origin for pelvic serous cancer. Clin Med Res 2007;5:35-44.

25. Medeiros F, Muto MG, Lee Y, et al. The tubal fimbria is a preferred site for early adenocarcinoma in women with familial ovarian cancer syndrome. Am J Surg Pathol 2006;30:230-236.

26. Antoniou A, Pharoah PD, Narod S, et al. Average risks of breast and ovarian cancer associated with BRCA1 or BRCA2 mutation detected in case series unselected for family history: A combined analysis of 22 studies. Am J Hum Genet 2003;72:1117-1130.

27. Risch HA, McLaughlin JR, Cole DE, et al. Prevalence and penetrance of germline BRCA1 and BRCA2 mutation in a population series of 549 women with ovarian cancer. Am J Hum Genet 2001;68:700-710.

28. Olopade OI, Artioli G. Efficacy of risk-reducing salpingo-oophorectomy in women with BRCA-1 and BRCA-2 mutations. Breast J 2004;10:S5-S9.

29. Eisen A, Lubinski J, Klijn J, et al. Breast cancer risk following bilateral oophorectomy in BRCA1 and BRCA2 mutation carriers: An international case-control study. J Clin Oncol 2005;23:74917496.

30. Marques RT, Baggerly KA, Patterson AP, et al. Patterns of gene expression in different histotypes of epithelial ovarian cancer correlate with those in the normal fallopian tube, endometrium, and colon. Clin Cancer Res 2005;11:6116-6126.

31. Colgan TJ, Boerner SL, Murphy J, Cole C, Narod S, Rosen B. Peritoneal lavage cytology: An assessment of its value during prophylactic oophorectomy. Gynecol Oncol 2002;85:397-403.

32. Sharifi S, Ducatman BS, Wang HH, Fraser JL. Peritoneal washing cytology is unnecessary in gynecologic surgery for benign diseases. Cancer 1999;87:259-262. 